\title{
Olmesartan - associated sprue-like enteropathy and colagenous colitis: A case report and review of literature
}

\author{
Nivedita Sudhekar ${ }^{1}$, Abrar Ahmed $^{2}$, Mark Feldman*1,2 \\ ${ }^{1}$ Department of Internal Medicine, Texas Health Presbyterian Hospital, Dallas, Texas, United States \\ ${ }^{2}$ Division of Gastroenterology, Texas Health Presbyterian Hospital, Dallas, Texas, United States
}

Received: January 19, 2019

DOI: $10.5430 /$ crim.v6n1p26
Accepted: February 14, 2019 Online Published: February 20, 2019

URL: https://doi.org/10.5430/crim.v6n1p26

\begin{abstract}
Olmesartan, an angiotensin II receptor blocker, is widely used for the treatment of hypertension. An association of olmesartan use with a reversible, sprue-like enteropathy has recently been recognized. There are also rare reports of olmesartan use and the combination of sprue-like enteropathy and collagenous colitis. We report a case of an elderly female who presented with both severe collagenous sprue and collagenous colitis associated with the use of olmesartan, with complete clinical and histologic recovery after discontinuation of the drug.
\end{abstract}

Key Words: Angiotensin receptor blocker, Olmesartan, Chronic diarrhea, Sprue-like enteropathy, Collagenous sprue, Intraepithelial lymphocytosis, Villous atrophy, Collagenous colitis

\section{INTRODUCTION}

Olmesartan medexomil is a prodrug of olmesartan, an angiotensin II receptor blocker (ARB). The drug is approved for the treatment of hypertension. ARBs such as olmesartan are also commonly used to enhance survival in patients with heart failure with reduced ejection fraction and to reduce proteinuria from diabetic or hypertensive nephropathy.

Recent reports have suggested an association between use of olmesartan and a sprue-like enteropathy, with affected patients presenting with severe, protracted diarrhea and weight loss that improves after discontinuation of olmesartan. ${ }^{[1-18]}$ Although the symptoms and histologic findings of olmesartan-associated enteropathy (OAE) resemble severe celiac disease, negative laboratory testing for celiac disease and lack of a response to a gluten-free diet suggest that OAE is a separate clinical entity. The pathogenesis of this entity remains to be elucidated. The most frequently reported histopathological findings in patients with OAE are villous architectural distortion and collagenous sprue in duodenal biopsies. There are rare cases of an associated microscopic colitis and sometimes collagenous colitis. ${ }^{[1,3,9-14]}$

We report a case of an elderly female taking olmesartan who presented with both severe sprue-like enteropathy and collagenous colitis which resolved clinically and histologically after discontinuation of this drug.

\section{Case representation}

A 70-year-old Caucasian female with hypertension receiving olmesartan $40 \mathrm{mg} /$ day for the past 7 months presented with 1 month of profuse, non-bloody, watery diarrhea. The diarrhea

\footnotetext{
*Correspondence: Mark Feldman; Email: MarkFeldman@texashealth.org; Address: Department of Internal Medicine, Texas Health Presbyterian Hospital, Dallas, Texas, 75231, United States.
} 
had a sudden onset and had worsened over the prior month with up to 10-15 stools per day, usually worse after meals and sometimes associated with vomiting. During this time, she had lost $6 \mathrm{~kg}$ of weight. There was no abdominal pain or tenesmus. She was unable to work. She denied recent travel, sick contacts, dietary changes, and use of over the counter supplements or herbal medicines. In addition to olmesartan, she was taking duloxetine $60 \mathrm{mg}$ daily for fibromyalgia and zolpidem $5 \mathrm{mg}$ at night as needed for insomnia. She had tried diphenoxylate/atropine to control her diarrhea but discontinued it due to abdominal cramps. She consulted her primary care provider after one week of diarrhea and underwent negative stool testing for bacterial pathogens. She also had had two emergency room visits in the past month due to volume depletion attributed to her diarrhea and vomiting. There she received volume resuscitation with intravenous lactated Ringer's solution. She presented to our outpatient gastroenterology clinic for further evaluation one month after the onset of her symptoms. She was still taking the olmesartan.

Her temperature was $97.8^{\circ} \mathrm{F}$ with a blood pressure of $88 / 68$ $\mathrm{mmHg}$, pulse 106 beats per min and respiratory rate 18 per minute. The physical examination revealed a fatigued female with no other focal findings. Her abdomen was soft, nondistended and non-tender without hepatosplenomegaly or ascites. Anorectal examination showed no hemorrhoids or fissures and a fecal occult blood test was negative. Laboratory tests for infectious and non-infectious causes of enteropathy, including immunoglobulin-A anti-tissue transglutaminase (IgA-tTG) antibody were negative. HIV testing was also negative. Complete blood count, basic metabolic panel, serum TSH and free thyroxine levels, and serum iron, ferritin, B12, and folate were normal.

Esophagogastroduodenoscopy showed a normal esophagus and stomach. There was slight mucosal scalloping with a nonspecific loss of the normal vascular pattern in the duodenum (see Figure 1). Biopsies of the duodenum showed sprue-like enteropathy with marked villous atrophy and flattening of the villi, chronic inflammation of the lamina propria with intraepithelial lymphocytosis and a thickened sub-epithelial collagen layer (see Figure 2). At colonoscopy the colon and rectum appeared normal. However, random colonic biopsies revealed collagenous colitis with a thickened sub-epithelial collagen layer (see Figure 3).

Discontinuation of olmesartan led to gradual clinical resolution of her symptoms and after 9 weeks she had complete resolution. No dietary restrictions or other medications were needed to control her symptoms. Duodenal and colonic biopsies obtained 5 months after discontinuing olmesartan were normal.

Published by Sciedu Press

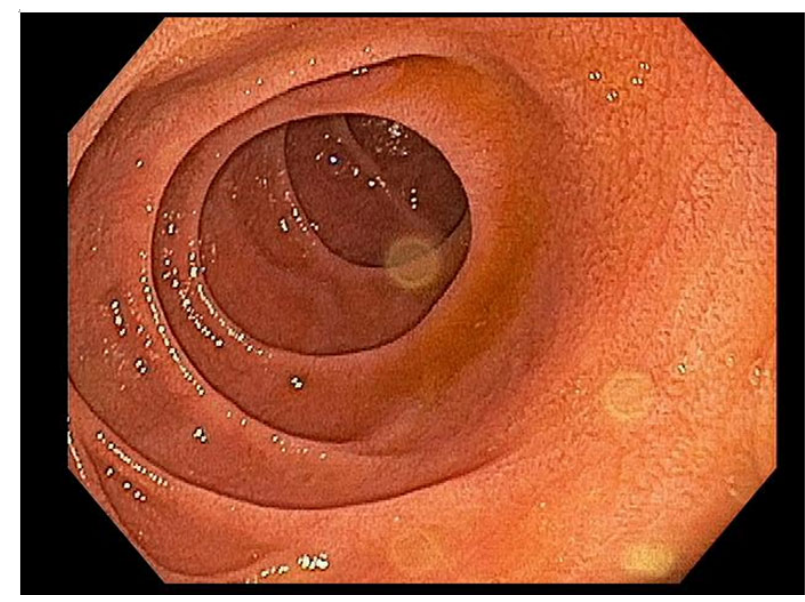

Figure 1. Duodenoscopy showed non-specific loss of vascular pattern and slight mucosal scalloping.

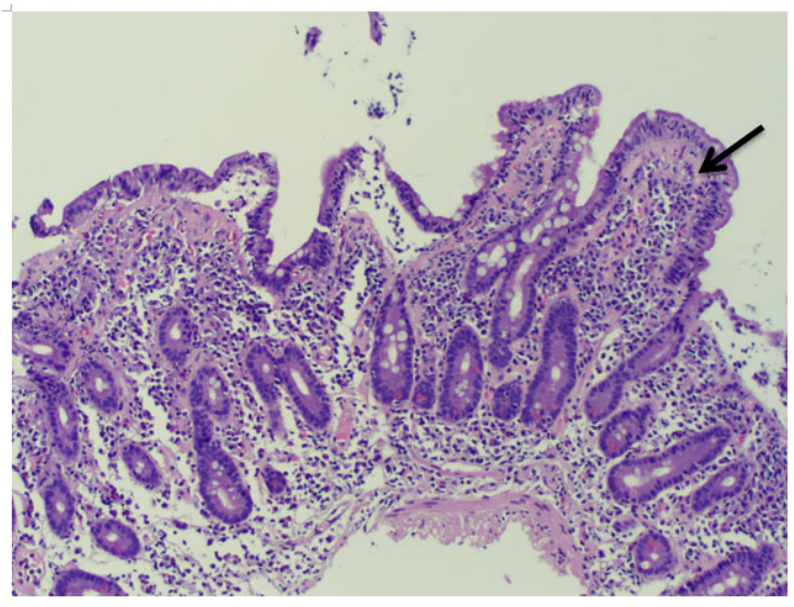

Figure 2. Duodenal mucosal biopsy showed variable villous blunting, lymphocytosis in the intraepithelial region and the lamina propria and increased sub-epithelial collagen (collagenous sprue; see arrow).

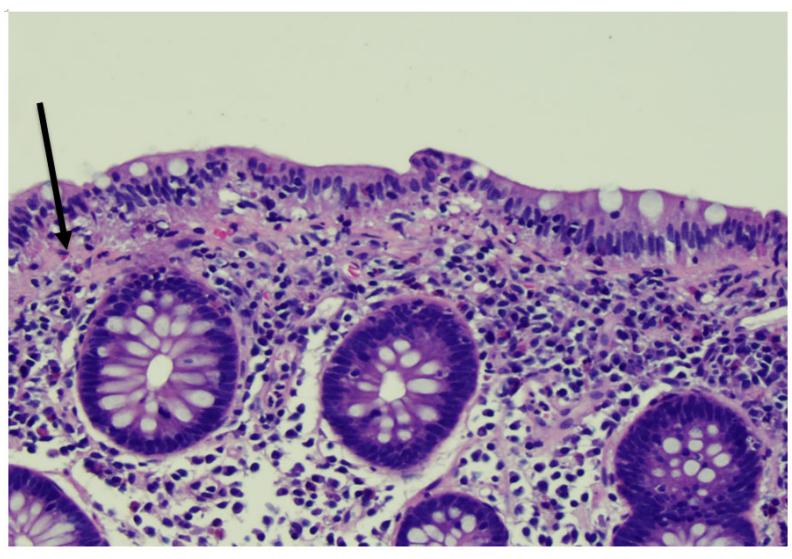

Figure 3. Colonic biopsy showed marked lymphocytic infiltration and a thickened apical sub-epithelial collagen layer (collagenous colitis; see arrow). The endoscopic appearance of the colon was normal. 


\section{DisCussion}

Olmesartan has been increasingly recognized to rarely cause a sprue-like enteropathy with villous atrophy and mucosal inflammation on small intestinal biopsies. ${ }^{[3,4,12,13]} \mathrm{OAE}$ affects men and women equally, with most affected patients between the age of 46-91 years (mean age, 68 years). ${ }^{[1-7]}$ The average dose of olmesartan in affected patients is 40 $\mathrm{mg}$ per day. Chronic diarrhea with weight loss is the most common clinical presentation. In a systematic review, other reported symptoms include fatigue $(56 \%)$, nausea and vomiting (45\%), abdominal pain (37\%) and bloating (29\%). ${ }^{[3]}$

Sprue-like enteropathy is characterized by diarrhea, villous atrophy of the small bowel mucosa with lymphocytic infiltration, intestinal malabsorption, weight loss and negative celiac disease serology testing. ${ }^{[1-4]}$ This entity remains a challenge due to a wide differential diagnosis including celiac disease, Crohn disease, drug-induced enteropathy, intestinal lymphoma (e.g., enteropathy-associated T cell lymphoma), infections (e.g., giardiasis), post-infectious enteropathy, idiopathic collagenous sprue, tropical sprue, autoimmune enteropathy, and enteropathy associated with some immunodeficiency disorders such as common variable immunodeficiency disorder, X-linked agammaglobulinemia, transplant-associated enteropathy and enteropathy associated with HIV infection. Other drugs that are implicated include neomycin, colchicine, chemotherapeutic agents (e.g., capecitabine), NSAIDs (e.g., sulindac), immunosuppressive agents such as methotrexate, azathioprine, and mycophenolate mofetil, and biological agents such as ipilimumab (CTLA-4 antibody) and etanercept (TNF inhibitor). ${ }^{[18]}$

In 2012, Rubio-Tapia et al were the first to describe 22 patients referred to the Mayo Clinic with severe, nonceliac sprue-like enteropathy attributed to olmesartan use. ${ }^{[1]}$ Olmesartan-associated enteropathy (OAE) can develop months to years after the initiation of this drug. Ianiro et al noted a 72\% prevalence of the HLA DQ2 or DQ8 haplotype in patients with $\mathrm{OAE}$, suggesting a genetic predilection. ${ }^{[3]}$

The pathophysiology of this entity remains to be elucidated. OAE is most likely an immune-mediated condition, probably involving delayed cell-mediated hypersensitivity. Olmesartan medoximil is converted to the active drug olmesartan during intestinal absorption. Whether the intestinal inflammatory response is to the prodrug (olmesartan medoximil), olmesartan itself, or a metabolite of olmesartan is unknown and there is no allergy testing available.

In addition to its vasopressor effect and its stimulatory effect on aldosterone secretion, angiotensin II, acting on AT1 receptors, increases intestinal growth factors, whereas angiotensin II acting on AT2 receptors induces apoptosis of enterocytes by up-regulation of pro-apoptotic proteins such as Bax and GATA-6 and by down-regulation of anti-apoptotic proteins such as Bcl-2. ${ }^{[14]}$ Olmesartan is selective for AT1 receptors over AT2 receptors. Olmesartan- induced selective AT1 receptor blockade also results in a translocation of AT2 receptors to the outer part of the basolateral cell membrane. ${ }^{[14,15]}$ This receptor translocation favors the binding of angiotensin II to AT2 receptors, possibly resulting in the pro-apoptotic effect seen in OAE. The duodenal-jejunal histology is characterized by villous atrophy and increased intra-epithelial lymphocytes (CD3 and CD8 lymphocytes) apparently due to apoptosis of enterocytes. ${ }^{[14,15]}$ Increased sub-epithelial collagen deposition (collagenous sprue) can also be seen.

Another study has proposed roles for IL-15 signaling and disruption of the tight junction protein zona occludens-1 (ZO-1) in disease pathogenesis and showed an overlap between the changes observed in OAE and patients with active and refractory celiac disease. ${ }^{[19]}$ There have also been observations that OAE patients exhibits varying degrees of granulocytic infiltration (with both neutrophils and eosinophils) and increased crypt cell apoptosis. ${ }^{[3,4,7,13,14,17]}$

There is no specific finding to establish the diagnosis of olmesartan-induced intestinal injury based solely on histopathology. Proving a cause-and-effect relationship requires discontinuation of olmesartan with complete resolution of OAE and then re-challenge with olmesartan medoxomil. Rechallenge is not usually performed given the severity of illness and availability of alternatives to olmesartan medoxomil. Gallivan and Brown et al reported a patient with severe diarrhea whose clinical symptoms and signs resolved upon discontinuation of olmesartan. Upon selectively reintroducing olmesartan, the patient had a recurrence of diarrhea. ${ }^{[13]}$ A similar case was reported by DeGaetani et al. ${ }^{[2]}$

Olmesartan medoximil has a longer half-life than other ARBs, possibly contributing to its association with enteropathy. Whether this association is specific for olmesartan medoximil or is a class effect of angiotensin II receptor blockers remains to be elucidated. Twelve cases have been reported with other ARBs such as irbesartan, valsartan, losartan, eprosartan and telmisartan. ${ }^{[7,8,16,20]}$ A cause-and-effect association of these other ARBs is not certain, however. A nationwide case-control study in Sweden failed to show an association of sprue-like enteropathy and use of either angiotensin converting enzyme inhibitors (ACEIs) or nonolmesartan ARBs. ${ }^{[15]}$ Also, a nationwide epidemiological study in France concluded that enteropathy associated with sartans other than olmesartan was a very rare occurrence. ${ }^{[4]}$

Histological recovery from OAE requires several months 
from the date of discontinuation of olmesartan. ${ }^{[1-4]}$ This was the case in our patient, in whom full histologic recovery was documented 5 months after discontinuing olmesartan medoximil.

Approximately 150 cases of olmesartan induced sprue-like enteropathy have now been described and the label of olmesartan has been changed to reflect this adverse event. ${ }^{[9]}$ However, only 7 cases of an associated collagenous colitis (CC) have been reported to-date in the literature. The prior 6 cases and our present case are summarized in Table 1. In our review of the literature, there has yet to be a reported case of collagenous colitis associated with olmesartan use without associated sprue-like enteropathy. The histopathological manifestations of collagenous colitis were similar to collagenous sprue, with colon biopsies showing increased intraepithelial lymphocytes, subepithelial collagen thickening, chronic inflammation of the lamina propria extending down to the crypt bases, and destruction of crypt epithelium. ${ }^{[14]}$

Table 1. Characteristics of reported cases of olmesartan-associated enteropathy with collagenous colitis

\begin{tabular}{lllllll}
\hline \multirow{2}{*}{ Author } & $\begin{array}{l}\text { Total no of } \\
\text { patients } \\
\text { with OAE } \\
\text { in the study }\end{array}$ & $\begin{array}{l}\text { Average } \\
\text { age, } \\
\text { years } \\
\text { (range) }\end{array}$ & $\begin{array}{l}\text { Average } \\
\text { olmesartan dose } \\
\text { mg/day } \\
\text { (range) }\end{array}$ & $\begin{array}{l}\text { Average olmesartan } \\
\text { duration } \\
\text { years } \\
\text { (range) }\end{array}$ & $\begin{array}{l}\text { Number of OAE } \\
\text { patients with } \\
\text { collagenous } \\
\text { colitis }\end{array}$ & $\begin{array}{l}\text { Sex of OAE } \\
\text { patients with } \\
\text { collagenous } \\
\text { colitis }\end{array}$ \\
\hline Rubio-Tapia $^{[1]}$ & 22 & $\begin{array}{l}69.5 \\
(47-81)\end{array}$ & $\begin{array}{l}30 \\
(10-40)\end{array}$ & $\begin{array}{l}3.1 \\
(0.5-7)\end{array}$ & 3 & $\mathrm{~F}, \mathrm{~F}, \mathrm{~F}$ \\
De Foneska $^{[21]}$ & 1 & 60 & 40 & 7 & 1 & $\mathrm{M}$ \\
Ebrahim $^{[22]}$ & 3 & 66 & 40 & 1.5 & 2 & $\mathrm{M}, \mathrm{M}$ \\
Our patient & 1 & $(57-72)$ & $(0.1-2)$ & 0.7 & 1 & $\mathrm{~F}$ \\
\hline
\end{tabular}

\section{Conclusion}

We report a case of olmesartan induced sprue-like enteropathy associated with collagenous colitis. It is important for physicians to become aware of the association of olmesartan use with OAE and CC. OAE/CC may mimic refractory celiac disease as well as idiopathic lymphocytic colitis. Some patients are misdiagnosed with celiac disease and initiated on a trial of a gluten-free diet, which delays definitive treatment for OAE (i.e olmesartan discontinuation). Some affected patients have also been treated with glucocorticoids for suspected inflammatory bowel disease before OAE was recognized. More cases like ours are expected as ARBs are being more commonly used for hypertension and heart failure with reduced ejection fraction.

\section{CONFLicts OF INTEREST Disclosure}

The authors have declared no conflicts of interest.

\section{REFERENCES}

[1] Rubio-Tapia A, Herman ML, Ludvigsson JF, et al. Severe spruelike enteropathy associated with olmesartan. Mayo Clin Proc. 2012; 87: 732-738. PMid:22728033. https://doi.org/10.1016/j . mayo cp. 2012.06 .003

[2] DeGaetani M, Tennyson CA, Lebwohl B, et al. Villous atrophy and negative celiac serology: a diagnostic and therapeutic dilemma. Am J Gastroenterol. 2013; 108: 647-653. PMid:23644957. https : //doi.org/10.1038/ajg.2013.45

[3] Ianiro G, Bibbo S, Montalto M, et al. Systematic review: Sprue-like enteropathy associated with olmesartan. Aliment Pharmacol Ther. 2014; 40: 16-23. https://doi.org/10.1111/apt. 12780

[4] Marthey L, Cadiot G, Seksik P, et al. Olmesartan-associated enteropathy: results of a national survey. Aliment Pharmacol Ther. 2014; 40: 1103-9. https://doi.org/10.1111/apt.12937

[5] Scialom S, Malamut G, Meresse B, et al. Gastrointestinal disorder associated with olmesartan mimics autoimmune enteropathy. PLoS
One. 2015; 10: e0125024. PMid:26101883. https ://doi .org/10 .1371/journal.pone. 0125024

[6] Hujoel IA, Rubio-Tapia A. Sprue-like enteropathy associated with olmesartan: a new kid on the enteropathy block. GE Port J Gastroenterol. 2016; 23: 61-65. PMid:28868435. https://doi .org/10.1 $016 / j \cdot j$ pge .2016 .02 .005

[7] Basson M, Mezzarobba M, Weill A, et al. Severe intestinal malabsorption associated with olmesartan: a French nationwide observational cohort study. Gut. 2016 Oct; 65(10): 1664-9. PMid:26250345. https://doi.org/10.1136/gutjnl-2015-309690

[8] Padwal R, Lin M, Etminan M, et al. Comparative effectiveness of olmesartan and other angiotensin receptor blockers in diabetes mellitus: retrospective cohort study. Hypertension. 2014; 63: 977-983. PMid:24535009. https://doi.org/10.1161/HYPERTENSIONAH A. 113.02855

[9] FDA. FDA Drug Safety Communication: FDA approves label changes to include intestinal problems (sprue-like enteropathy) linked 
to blood pressure medicine olmesartan medoxomil. 2013. Available from: www.fda.gov/Drugs/DrugSaf ety/ucm359477.htm

[10] Galanopoulos M, Varytimiadis L, Tsigaridas A, et al. Small bowel enteropathy associated with olmesartan medoxomil treatment. Ann Gastroenterol. 2017; 30: 131-133. https://doi.org/10.20524 /aog. 2016.0052

[11] Bhat N, Anupama NK, Yelsangikar A, et al. Olmesartan-related sprue-like enteropathy. Indian J Gastroenterol. 2014; 33: 564 567. PMid:25303875. https : //doi .org/10.1007/s12664-014 -0506-7

[12] Dreifuss SE, Tomizawa Y, Farber NJ, et al. Spruelike enteropathy associated with olmesartan: an unusual case of severe diarrhea. Case Rep Gastrointest Med. 2013; 2013: 618071. https : //doi.org/10.1155/2013/618071

[13] Gallivan C, Brown I. Olmesartan induced enterocolitis. Pathology. 2014; 46: 360-361. PMid:24798161. https://doi.org/10.109 7/PAT. 0000000000000104

[14] Choi EY, McKenna BJ. Olmesartan-associated enteropathy: a review of clinical and histologic findings. Arch Pathol Lab Med. 2015; 139 1242-7. PMid:26414468. https : //doi .org/10.5858/arpa. 201 5-0204-RA

[15] Marild K, Lebwohl B, Green PH, et al. Blockers of angiotensin other than olmesartan in patients with villous atrophy: a nationwide casecontrol study. Mayo Clin Proc. 2015; 90: 730-737. PMid:26046408. https://doi.org/10.1016/j.mayocp.2015.04.002

[16] Mandavdhare HS, Sharma V, Prasad KK, et al. Telmisartan-induced sprue-like enteropathy: a case report and a review of patients using non-olmesartan angiotensin receptor blockers. Intest Res. 2017; 15:
419-421. PMid:28670240. https ://doi.org/10.5217/ir. 201 7.15 .3 .419

[17] Fandriks L. The angiotensin II type 2 receptor and the gastrointestinal tract. J Renin Angiotensin Aldosterone Syst. 2010; 11: 43-8. PMid:19861352. https ://doi.org/10.1177/14703203093477 88

[18] Freeman HJ. Drug-induced sprue-like intestinal disease. International Journal of Celiac Disease. 2014; 2(2): 49-53. https : //doi.org/ 10.12691/ijcd-2-2-5

[19] Marietta EV, Nadeau AM, Cartee AK, et al. Immunopathogenesis of olmesartan-associated enteropathy. Aliment Pharmacol Ther. 2015 Dec; 42(11-12): 1303-14. https://doi.org/10.1111/apt.13 413

[20] Zanelli M, Negro A, Santi R, et al. Letter: sprue-like enteropathy associated with angiotensin II receptor blockers other than olmesartan Aliment Pharmacol Ther. 2017. https://doi.org/10.1111/apt . 14176

[21] de Fonseka A, Tuskey A, Moskaluk C. A case of olmesartan induced enteropathy. Inflamm Bowel Dis. 2012; 18: S17. https: //doi.org/10.1097/00054725-201212001-00043

[22] Ebrahim VS, Martin J, Murthy S, et al. Olmesartan-associated enteropathy. Proc (Bayl Univ Med Cent). 2017 Jul; 30(3): 348-350. PMid:28670083. https://doi.org/10.1080/08998280.2017. 11929644

[23] Adike A, Corral J, Rybnicek D, et al. Olmesartan-induced enteropathy. Methodist Debakey Cardiovasc J. 2016 Oct-Dec; 12(4): 230-232. https : //doi .org/10.14797/mdcj-12-4-230 